\title{
Impact of oral galenic formulations of Lactobacillus salivarius on probiotic survival and interactions with microbiota in human in vitro gut models
}

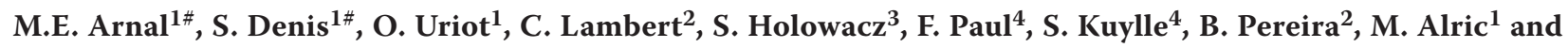 \\ S. Blanquet-Diot ${ }^{1^{*}}$
}

${ }^{1}$ Université Clermont Auvergne, UMR 454 MEDIS, Microbiologie Environnement Digestif et Santé, 28 place Henri Dunant, 63000 Clermont-Ferrand, France; ${ }^{2}$ University Hospital Clermont-Ferrand, Biostatistics Units, 58, rue Montalembert, 63000 Clermont-Ferrand, France; ${ }^{3}$ PiLeJe Industrie, Parc Naturopôle, Les Tiolans 03800 Saint-Bonnet de Rochefort, France; ${ }^{4}$ Genibio, Le Pradas, ZI du Couserans, 09190 Lorp-Sentaraille, France; stephanie.blanquet@uca.fr; ${ }^{*}$ these authors contributed equally

Received: 17 September 2020 / Accepted: 16 March 2020

(c) 2021 Wageningen Academic Publishers

OPEN ACCESS (1) (1)(2) RESEARCH ARTICLE

\begin{abstract}
Health benefits of probiotics in humans essentially depend on their ability to survive during gastrointestinal (GI) transit and to modulate gut microbiota. To date, there is few data on the impact of galenic formulations of probiotics on these parameters. Even if clinical studies remain the gold standard to evaluate the efficacy of galenic forms, they stay hampered by technical, ethical and cost reasons. As an alternative approach, we used two complementary in vitro models of the human gut, the TNO gastrointestinal (TIM-1) model and the Artificial Colon (ARCOL), to study the effect of three oral formulations of a Lactobacillus salivarius strain (powder, capsule and sustained-release tablet) on its viability and interactions with gut microbiota. In the TIM-1 stomach, no or low numbers of bacteria were respectively released from the capsule and tablet, confirming their gastro-resistance. The capsule was disintegrated in the jejunum on average $76 \mathrm{~min}$ after administration while the core of sustained-release tablet was still intact at the end of digestion. Viability in TIM-1 was significantly influenced by the galenic form with survival percentages of $0.003 \pm 0.004 \%, 2.8 \pm 0.6 \%$ and $17.0 \pm 1.8 \%(\mathrm{n}=3)$ for powder, capsule and tablet, respectively. In the ARCOL, the survival of the strain tended to be higher in the post-treatment phase with the tablet compared to capsule, but gut microbiota composition and activity were not differently modulated by the two formulations. In conclusion, the sustained-release tablet emerged as the formulation that most effectively preserved viability of the tested strain during GI passage. This study highlights the usefulness of in vitro gut models for the pre-screening of probiotic pharmaceutical forms. Their use could also easily be extended to the evaluation of the effects of food matrices and age on probiotic survival and activity during GI transit.
\end{abstract}

Keywords: lactic acid bacteria, oral galenic formulations, in vitro gut model, viability, gut microbiota

\section{Introduction}

Probiotics are defined as 'live microorganisms that, when administered in adequate amounts, confer a health benefit on the host' (FAO/WHO, 2002; Hill et al., 2014). They have been shown to improve host's health through various mechanisms of action, including competitive exclusion of pathogens and bacteriocin production, modulation of faecal enzymatic activities associated with the metabolisation of biliary salts and inactivation of carcinogens, production of short-chain and branched-chain fatty acids, cell adhesion and mucin production, modulation of the immune system, and interaction with the brain-gut axis by regulation of endocrine and neurological functions (He and Shi, 2017; Plaza-Diaz et al., 2019). More and more studies are highlighting the central role of gut microbiota modulation by probiotics in such beneficial effects (Azad et al., 2018). Among available microorganisms, strains of lactic acid bacteria belonging to the genera Lactobacillus and Bifidobacterium are commonly used as probiotics. In particular, Lactobacillus salivarius strains, which have been frequently isolated from human, porcine and avian gastrointestinal tracts (GIT) and human 
milk, are well-characterised producers of antimicrobial substances, such as bacteriocin. In recent years, several L. salivarius strains have gained attention as promising probiotics due to their ability to modulate gut microbiota by modifying the ratio of different commensal lactic acid and other bacteria, produce short chain fatty acids (SCFA) allowing an advisable acidification of the gut, and stimulate protective immune response, among others (Chaves et al., 2017; Messaoudi et al., 2013).

Once in the human digestive environment, probiotics have to face various stresses, including gastric acid $\mathrm{pH}$, bile salts and enzymes, and then compete for nutrients and niches with resident microbiota. According to the legal definition, survival of probiotics during transit through the host GIT is a key feature for their activity. Probiotic survival, as well as their beneficial effects, are assumed to be largely straindependent (Campana et al., 2017; Marteau et al., 1997). Probiotic survival in the human gut will not only depend on the strains, but also on their mode of administration, i.e. under fed or fasted state and with different food matrices (Blanquet-Diot et al., 2012; Soares et al., 2019; Vijaya Kumar et al., 2015). Survival is also related to the host environment, i.e. different ages or healthy or pathological situations (Sniffen et al., 2018). Ideally, probiotic strains should exhibit a natural tolerance to gastric and bile acids, as well as sufficient resistance against digestive enzymes which favours their survival during the passage through stomach and upper intestinal tract (Venema et al., 2019). As it is frequently not the case, diverse strategies that protect probiotics against gastric acidity can be applied, such as microencapsulation (Surono et al., 2018) and enteric coating of tablets (Eiberger et al., 2011). Specific formulations have been also designed to target specific sites of action in the human GIT, such as sustained-release formulations (Gately and Kennedy, 2017).

Clinical studies are the gold standard to evaluate probiotic survival and activity in the human gut. Nevertheless, such approaches are hampered by regulatory, technical and cost reasons and are often limited to end-point measurements in human faecal samples. As a relevant complementary approach to in vivo assays, in vitro gut models can be advantageously used to get insights into probiotic survival and mode of action in all human digestive compartments, especially in relation with gut microbiota interactions. Among available models, the well-validated dynamic systems, such as the TNO gastrointestinal model (TIM-1), the Simulator of the Human Microbial Ecosystem (SHIME) and the Artificial colon (ARCOL) are those that are more closely reproducing in vivo conditions. They have been successfully used for probiotic testing (Blanquet-Diot et al., 2012; Cordonnier et al., 2015; Patrignani et al., 2019; Thévenot et al., 2015; Van den Abbeele et al., 2012; Venema et al., 2020, 2019). In the present study, we used two complementary dynamic in vitro models, TIM-1 which simulates the human stomach and three parts of the small intestine and ARCOL which reproduces the human colon, to assess the impact of three galenic formulations (powder, capsule and sustained-release tablet) of a $L$. salivarius strain on its survival all along the gut and interactions with resident gut microbiota. Its viability was investigated by plating or by propidium monoazide quantitative PCR (PMAqPCR) analysis. Gut microbiota composition was assessed through Illumina 16S Miseq sequencing and microbial activities by measurement of short chain fatty acids and gases.

\section{Materials and methods}

\section{Bacterial probiotic strain and galenic formulations}

The $L$. salivarius strain (registered under the number CNCM I-4973) was supplied by Pileje Industrie (SaintBonnet de Rochefort, France) (Holowacz et al., 2018). Three oral galenic formulations based on the dehydrated strain were tested. The powder form contained corn maltodextrins and $L$. salivarius strain ( $125 \mathrm{mg}$ per $2 \mathrm{~g}$ ). The hydroxypropylmethylcellulose (HPMC) capsule (size 1, total 325 $\mathrm{mg}$ ) contained corn starch, hydroxypropylmethylcellulose, magnesium stearate and $L$. salivarius strain $(125 \mathrm{mg}$ ). The sustained-release tablet (total $350 \mathrm{mg}$ ) contained microcrystalline cellulose, di-calcium phosphate, hydroxypropylmethylcellulose, glycerides, magnesium stearate and L. salivarius strain (125 mg). Each powder sachet, capsule and tablet form contained $(7.6 \pm 0.5) \times 10^{9},(1.0 \pm 0.2) \times 10^{10}$ and $(5.0 \pm 0.7) \times 10^{9} \mathrm{cfu}$ of the $L$. salivarius strain, respectively (mean $\pm \mathrm{SD}, \mathrm{n}=5$ ).

\section{In vitro digestion protocols}

\section{Artificial digestions in the TIM-1 gastro-intestinal model}

The TIM-1 model (TNO, Zeist, the Netherlands) consists of four successive compartments simulating the human stomach and the three segments of the small intestine, duodenum, jejunum, and ileum. TIM-1 is a computercontrolled systems in which the main parameters of human digestion, such as body temperature, kinetics of gastric and intestinal $\mathrm{pH}$, peristaltic mixing and transport, gastric, biliary, and pancreatic secretions and passive absorption of small molecules and water, are reproduced as accurately as possible, based on in vivo data (Cordonnier et al., 2015; Roussel et al., 2016). Before each experiment, the system is decontaminated by steaming at $105{ }^{\circ} \mathrm{C}$ for $45 \mathrm{~min}$. In the present study, the TIM-1 system was programmed to reproduce the physicochemical conditions observed during the digestion of a glass of water (fasted state) in a healthy human adult, as previously described (Roussel et al., 2016, Supplementary Table S1). The three formulations were digested in the TIM-1 system with $200 \mathrm{ml}$ of naturally sterile mineral water. The powder formulation $(2 \mathrm{~g})$ was rehydrated and homogenised for $10 \mathrm{~min}$ in mineral water 
before introduction into the TIM-1 gastric compartment, while the capsule or tablet was placed in a pharmaceutical basket in the center of the gastric compartment before water was added. The pharmaceutical basket was manually transferred from one TIM-1 compartment to the following, after appropriate residence time in the gastric $(30 \mathrm{~min})$, duodenal (5 min), jejunal (90 $\mathrm{min})$ and ileal (115 min) compartments. In order to assess the strain survival, samples were regularly collected during digestion in the different compartments of the TIM-1 system, as well as in the cumulative ileal effluents kept on ice and pooled hourby-hour. The total duration of the experiments was $240 \mathrm{~min}$. At the end of the experiment, the gastrointestinal residue was collected from all compartments. Each condition was performed in triplicate. An overview of the experimental schedule in the TIM-1 system is given in Figure 1.

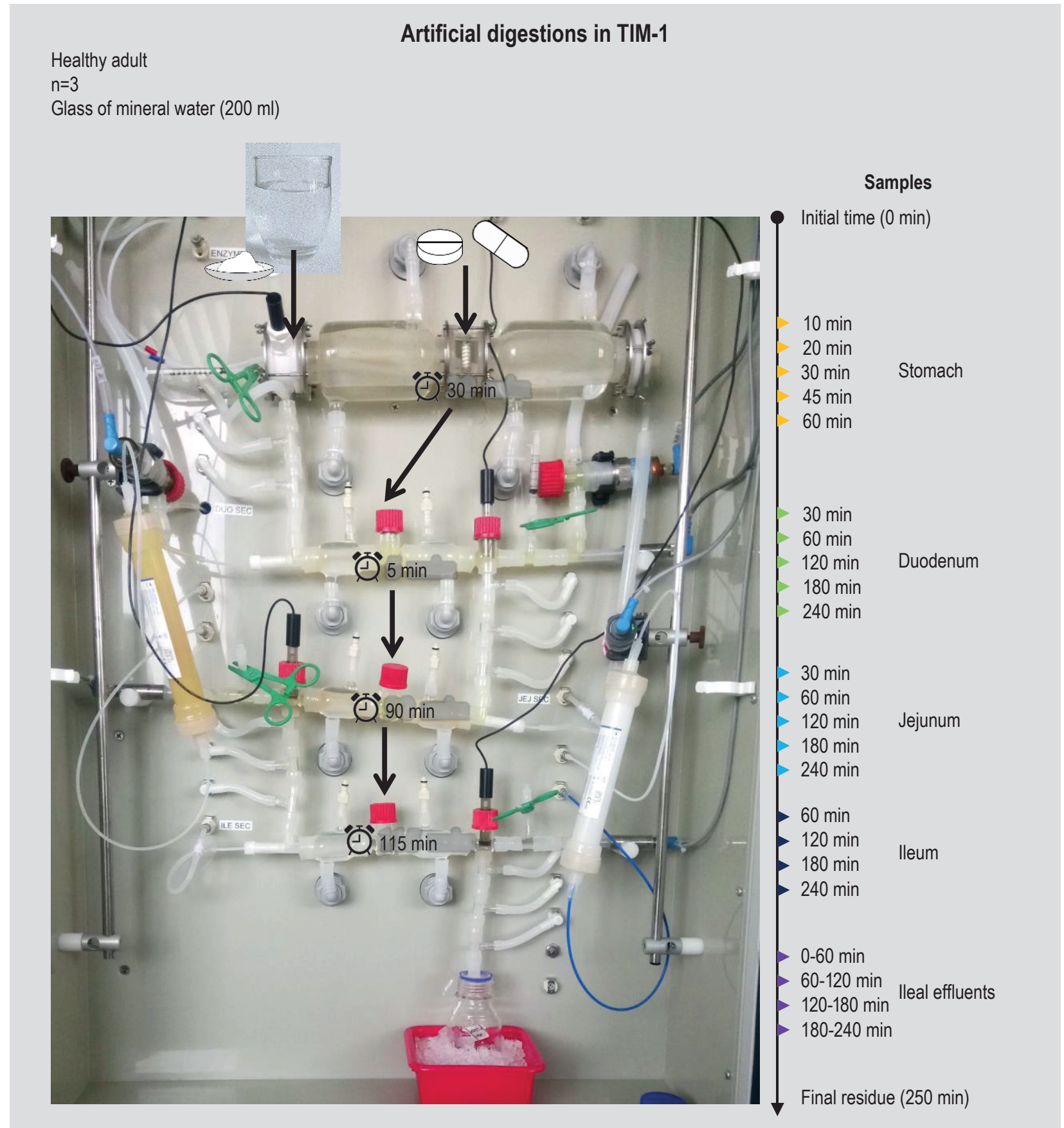

Figure 1. Overview of in vitro experiments. Two series of experimentations were performed to investigate in vitro the effect of three oral formulations of a Lactobacillus salivarius strain (powder, HPMC capsule and sustained-release tablet) on its survival throughout the human gastrointestinal tract and interactions with resident microbiota. Human gastric and small intestinal conditions were reproduced by the TIM-1 system while human colonic environment was simulated by the ARCOL model. 
Static batch gastrointestinal digestions

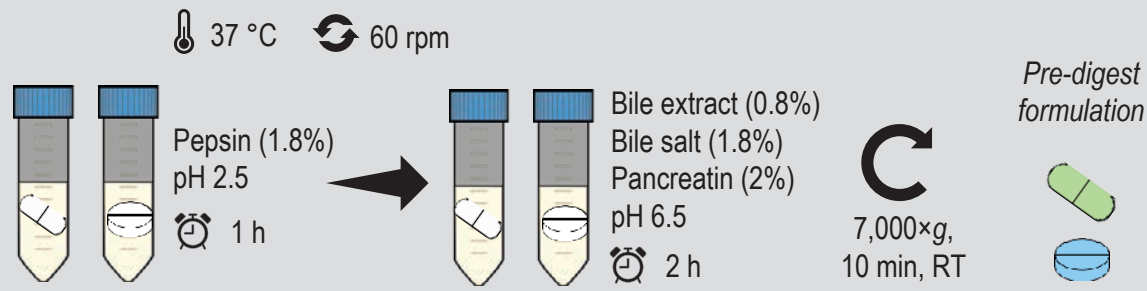

Artificial fermentations in ARCOL
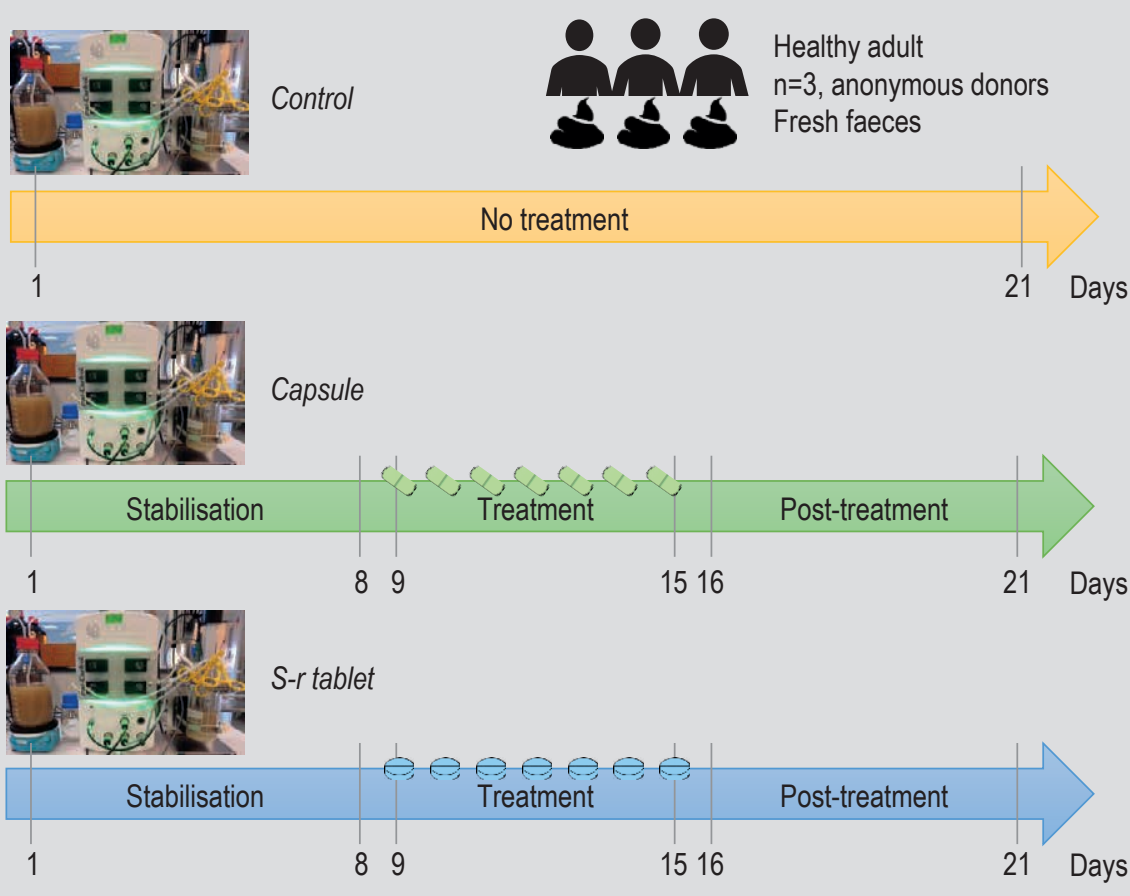

Figure 1. Continued.

Static batch gastrointestinal digestion

In order to reproduce gastro-intestinal stresses that probiotic encounter in the upper GIT, galenic formulations of the L. salivarius strain (capsule and tablet) were subjected to a static digestion protocol before introduction into the ARCOL model (to be noted: the TIM-1 and ARCOL models cannot be directly coupled) (Figure 1). Briefly, the procedure consisted of two successive steps simulating the mean conditions found in the human stomach and small intestine (Thévenot et al., 2015). Gastric environment was reproduced by addition of $1.8 \%(\mathrm{w} / \mathrm{v})$ of porcin pepsin (P7012, Sigma, St. Louis, MO, USA) to an aerobic culture of $L$. salivarius followed by acidification with $1 \mathrm{M} \mathrm{HCl}$ to $\mathrm{pH} 2.5$. The gastric phase was performed for $1 \mathrm{~h}$. Intestinal conditions were mimicked by addition of $1 \mathrm{M} \mathrm{NaHCO} 3$ up to $\mathrm{pH} 6.5$ followed by $2 \%, 1.6 \%$ and $0.8 \%(\mathrm{w} / \mathrm{v})$ of 4 USP porcine pancreatin (P1750, Sigma), bile salts for microbiology (mixture of sodium cholate and sodium deoxycholate, 48305, Fluka, Charlotte, NC, USA) and bile porcine extract (B8631, Sigma) respectively. The small intestinal phase was performed for $2 \mathrm{~h}$. All incubation steps were performed at $37^{\circ} \mathrm{C}$ under shaking $(60 \mathrm{rpm})$. After digestion, bacteria were harvested by centrifugation $(7,000 \times g, 10 \mathrm{~min}$, room temperature) and resuspended in sterile saline water before inoculation into the ARCOL model.

\section{Artificial fermentations in the ARCOL colon model}

Human colonic conditions were simulated in the ARCOL model using MiniBio $500 \mathrm{ml}$ my-Control bundles and Lucullus $^{\oplus}$ Lite software from Applikon (Applikon, Delft, the Netherlands). The ARCOL model integrates the main parameters of the in vivo human colonic environment (Cordonnier et al., 2015; Thévenot et al., 2015), including $\mathrm{pH}$, body temperature, supply of ileal effluents, retention time, anaerobiosis maintained by the sole activity of 
resident microbiota, and a complex and metabolically active resident colonic microbiota. Fresh faeces from three healthy adult volunteers who had no history of antibiotic or probiotic treatment 3 months before the study were used to inoculate the bioreactor (in order to perform three biological replicates). Faecal inocula for the in vitro colon model were prepared using $50 \mathrm{~g}$ of fresh faecal samples under strict anaerobic conditions in a vinyl anaerobic chamber (Coy, Grass Lake, MI, USA). Stools were mixed with $450 \mathrm{ml}$ of a $30 \mathrm{mM}$ anaerobic sodium phosphate buffer ( $\mathrm{pH}$ 6.5) supplemented with $1.9 \mathrm{mM}$ cysteine and the suspension was filtered through a double layer of gauze. The faecal suspension was divided into $100 \mathrm{ml}$ aliquots that were rapidly transferred into three bioreactors containing $200 \mathrm{ml}$ reduced nutritive medium flushed with $\mathrm{O}_{2}$-free $\mathrm{N}_{2}$ gas. Afterwards, during the fermentation course, the anaerobic conditions were maintained exclusively through the activity of the resident microbiota and by ensuring the airtightness of the system. The ARCOL model was run under mean conditions reproducing a healthy human colon. The temperature of the fermentation was set up to $37^{\circ} \mathrm{C}$. The $\mathrm{pH}$ was maintained to a value of 6.3 with an automatic addition of $2 \mathrm{M} \mathrm{NaOH}$. Redox potential was constantly measured using a redox sensor (Mettler Toledo, Viroflay, France). To allow biomass growth, a 24 h-batch fermentation was conducted after inoculation. Then, continuous fermentations were performed from day 2 until the end of experiment. They consisted in a constant introduction of nutritive medium into the bioreactor and a regular withdraw of fermentation medium ensuring a mean retention time of $24 \mathrm{~h}$ (with a reaction volume of $300 \mathrm{ml}$ ). After a 7 day stabilisation phase of the microbiota, the pre-digested oral formulations of L. salivarius (capsule and sustained-release tablet, powder was not tested) were administered once a day during 7 days in two of the three bioreactors (treatment period). The third bioreactor was the control condition with no probiotic treatment. Lastly, a 6-day post-treatment period where the probiotic was no longer added was conducted (see Figure 1 for experimental schedule). Samples were regularly collected during the 21 day experiment from the fermentation medium and atmospheric phase of the bioreactors, in order to determine probiotic survival, microbiota composition and microbial metabolic activities.

\section{Viability of the probiotic strain}

\section{Plate counts}

Survival kinetics of the probiotic L. salivarius strain was determined in each digestive compartment of the TIM-1 model by direct plating onto de Man, Rogosa and Sharpe (MRS) agar. Plates were incubated for 2 days at $37^{\circ} \mathrm{C}$ under anaerobiosis. Number of cultivable bacteria was determined by visual counting and viability results were expressed as $\log _{10} \mathrm{cfu}$.

\section{PMA-qPCR}

Samples from the fermentation medium were collected in duplicate from the ARCOL model and stained or not with $50 \mu \mathrm{M}$ PMA (Interchim, Montluçon, France). Samples were incubated $5 \mathrm{~min}$ at room temperature in the dark, under agitation (100 rpm). Then, stained samples were exposed 15 min to the blue light PMA-Lite LED photolysis (Interchim, Montluçon, France) to allow photo activation of the dye. Finally, both samples (stained or not) were centrifuged $\left(4,400 \times g, 4{ }^{\circ} \mathrm{C}, 10 \mathrm{~min}\right)$ and pellets were washed with milli-Q water and stored at $-20^{\circ} \mathrm{C}$ until DNA extraction. Total bacterial DNA from samples treated or not with PMA were extracted using the Smart Extract Kit SK-DNEX-100 (Eurogentec, Seraing, Belgium) in a single tube according to the manufacturer's instructions. Then, DNA were stored at $-20^{\circ} \mathrm{C}$. DNA concentration and purity were assessed by measuring the absorbance at $260 \mathrm{~nm}$ and the ratio of absorbance at 260 and $280 \mathrm{~nm}$ (A260/A280) with a NanoDrop ND-1000 spectrophotometer (Thermo Scientific, Waltham, MA, USA). qPCR was performed with L. salivarius specific primers and probe on a Stratagene Mx3005P qPCR system (Agilent Technologies, Massy, France). The total reaction volume of $10 \mu \mathrm{l}$ contained $5 \mu \mathrm{l}$ of Takyon Low Rox Probe Master Mix dTTP blue (Eurogentec, Seraing, Belgium), $0.2 \mu \mathrm{l}(10 \mu \mathrm{M})$ of each primer targeting L. salivarius (F-GCAGCAGTAGGGAATCTTC, R-CTCTCACTCGTGTTCTTCTC), $0.1 \mu \mathrm{l}$ of probe (CGCAAGTCTGATGGAGCAACGCC), $2.5 \mu$ l of water and $2 \mu \mathrm{l}$ of template DNA from colonic samples. Primers were specifically designed during the study to ensure an accurate tracking of the strain even with a complex microbial background like in the ARCOL model. Nontemplate control was $2 \mu \mathrm{l}$ milli-Q water. Each reaction was run in triplicate in a 96-well reaction plate sealed. The PCR reactions were amplified by an initial denaturation step at $95{ }^{\circ} \mathrm{C}$ for $5 \mathrm{~min}$ followed by 40 cycles of $95^{\circ} \mathrm{C}(30 \mathrm{~s})$, $60{ }^{\circ} \mathrm{C}(30 \mathrm{~s})$ and $72{ }^{\circ} \mathrm{C}(30 \mathrm{~s})$. The melting curves of PCR amplicons yielded a single melting peak were checked to ensure primer specificity.

\section{Gut microbiota activity}

\section{Gas}

Analysis of $\mathrm{O}_{2}, \mathrm{~N}_{2}, \mathrm{CO}_{2}, \mathrm{CH}_{4}$ and $\mathrm{H}_{2}$ found in the atmospheric phase of the bioreactor was performed daily using a 490 micro-gas chromatograph (Agilent Technologies, Santa Clara, CA, USA) equipped with two columns, Molecular Sieve 5A and PoraPlot U, coupled with TCD detectors. Argon was used as the carrier gas. Gas composition was determined using calibration curves made from ambient air $\left(78 \% \mathrm{~N}_{2}, 21 \% \mathrm{O}_{2}, 0.04 \% \mathrm{CO}_{2}\right)$ and three gas mixtures $\mathrm{A}\left(5 \% \mathrm{CO}_{2}, 5 \% \mathrm{H}_{2}, 90 \% \mathrm{~N}_{2}\right)$, B $(20 \%$ $\left.\mathrm{CO}_{2}, 80 \% \mathrm{H}_{2}\right)$ and $\mathrm{C}\left(20 \% \mathrm{CO}_{2}, 20 \% \mathrm{H}_{2}, 20 \% \mathrm{CH}_{4}, 40 \%\right.$ $\mathrm{N}_{2}$ ). Results were expressed in relative percentages. The 
daily total volume of gas overproduced was also measured using a gas sampling bag connected to the condenser of the bioreactors.

\section{Short chain fatty acids}

Samples collected daily from the ARCOL fermentative medium were centrifuged at $18,000 \times g$ for $15 \mathrm{~min}$ at $4{ }^{\circ} \mathrm{C}$ and supernatants were filtered $(0.45 \mu \mathrm{m})$. Concentrations of the three main SCFAs (acetate, propionate and butyrate) were determined using high performance liquid chromatography (HPLC) (Elite LaChrom, Merck Hitachi, Darmstadt, Germany) coupled with a diode-array detector. The HPLC column (ICSep ICE-COREGEL 87H3 $9 \mu \mathrm{m} 150 \times 7.8 \mathrm{~mm}$, Concise Separations, San Jose, CA, USA) and its guard column were maintained at $50{ }^{\circ} \mathrm{C}$. Sulfuric acid $0.008 \mathrm{~N}$ was used as mobile phase and SCFAs were separated at a flow rate of $0.6 \mathrm{ml} / \mathrm{min}$. Data was analysed by the EZChrom Elite software at $205 \mathrm{~nm}$. SCFA concentrations (expressed in relative percentages) were calculated from calibration curves established with known concentrations of acetate, propionate and butyrate $(0,10,25$ and $40 \mathrm{mM})$.

\section{Gut microbiota composition}

DNA was extracted from ARCOL fermentation medium at the end of each phase (stabilisation day 7, probiotic treatment day 13 and post-treatment phase day 21) with the QIAamp PowerFecal DNA Kit (Qiagen, Hilden, Germany) following manufacturer's instructions. DNA concentration and purity were assessed by measuring the absorbance at $260 \mathrm{~nm}$ and the ratio of absorbance at 260 and $280 \mathrm{~nm}$ (A260/A280) with a NanoDrop ND1000 spectrophotometer (Thermo Scientific, Waltham, MA, USA). DNA integrity was checked by agarose gel electrophoresis. Samples were stored at $-20^{\circ} \mathrm{C}$ prior to analysis. 16S rRNA gene Illumina MiSeq protocol was used for sequencing $(2 \times 250$ bp pair-end run, $10 \mathrm{M}-20 \mathrm{M}$ paired reads). The $\mathrm{V} 4$ variable region ( $400 \mathrm{bp})$ of the 16S rRNA gene was amplified (V4-515F-ACACTGACG ACATGGTTCTACAGTGYCAGCMGCCGCGGTAA, V4-806R-TACGGTAGCAGAGACTTGGTCTGGACTAC NVGGGTWTCTAAT). Amplicons were generated using a Fluidigm Access Array followed by high-throughput sequencing on an Illumina MiSeq system (Illumina, San Diego, CA, USA) performed at the Carver Biotechnology Center of the University of Illinois (Urbana, IL, USA). The demultiplexed paired end Illumina sequence reads in the FastQ format were uploaded into the Galaxy instance (v.2.3.0) of the Genotoul bioinformatics platform (http:// sigenae-workbench.toulouse.inra.fr) to be used in the FROGS (Find Rapidly OTU with Galaxy Solution) pipeline. During the FROGS pre-process, sequences were depleted of barcode and the sequences with a non-appropriate length or containing ambiguous bases were removed. Next, reads were clustered into de novo operational taxonomic units
(OTUs) using SWARM algorithm with, at first, a denoising step to build very fine cluster using the minimal distance equal to 1 and, secondly, with an aggregation distance equal to 3. Chimeras were then detected and removed with VSEARCH. Additionally, filters were applied to the OTUs in order to remove singletons. The OTUs selected were taxonomically assigned using the Silva release 132 reference database.

\section{Statistical analysis}

Statistical analysis was performed using R 3.4.3 (http:// cran.r-project.org/). All tests were two-sided, with a Type I error set at 0.05. Categorical parameters were expressed as frequencies and associated percentages, and continuous data as mean \pm standard deviation or as median [interquartile range], according to statistical distribution. In order to evaluate whether the evolution of bacteria amount over time was different according to the galenic form, linear mixed models were used considering the subject as a random effect. The dependent variable of the different models was the bacteria amount and the independent variables were the galenic form, the time and their interaction. The same analyses were performed for cumulative gas volumes in ARCOL.

\section{Results}

\section{Probiotic strain viability throughout the simulated human digestive tract}

\section{Gastric and small intestinal compartments}

In the TIM-1 gastric compartment, with the powder form, the number of cultivable bacteria decreased from $10 \log 10$ cfu at the initial time to $4 \log 10 \mathrm{cfu}$ at $60 \mathrm{~min}$ digestion due to a combined effect of both gastric emptying and low acid $\mathrm{pH}$ (Figure 2A). Gastric plotting for the probiotic was below the curve for the theoretical transit marker provided by the TIM-1 system clearly indicating bacterial mortality in this compartment (data not shown). With the sustained-release tablet, bacterial amounts ranging from $6 \log 10 \mathrm{cfu}$ at $10 \mathrm{~min}$ to $4 \log 10 \mathrm{cfu}$ at $60 \mathrm{~min}$ were found during gastric digestion. These decreasing counts resulted from the combination of progressive probiotic release from the tablet, emptying of the stomach compartment and probably bacterial mortality due to acid $\mathrm{pH}$. In any case, the amount of released bacteria at the very beginning of gastric digestion (before $\mathrm{pH}$ was too acidic) remained very low compared to tablet load $(9.5 \log 10)$. From 10 to $45 \mathrm{~min}$ of gastric digestion, bacterial amounts with the tablet were significantly lower than those found with the powder $(P<0.001)$. Lastly, no bacteria were found by plating over time for the HPMC capsule, showing its gastro-resistance $(P<0.001$ vs powder form). Whatever the galenic formulation, no viable bacteria was recovered in the 
duodenal compartment, suggesting the strong bactericidal effect of digestive secretions (bile salts and pancreatic juice). In the jejunal compartment, bacterial levels around $4 \log 10 \mathrm{cfu}$ were plated for the powder form. Compared to powder form, significantly higher amounts of probiotic were found during all the experiments (from 60 to $240 \mathrm{~min}$ ) with the capsule and the sustained-release tablet, with 7-8 and to 6-7 $\log 10 \mathrm{cfu}$, respectively $(P<0.001)$. On average, the complete disintegration of the capsule was observed in the jejunum at $76 \mathrm{~min}( \pm 5 \mathrm{~min})$ after administration. Lastly, in the ileal compartment, cultivable bacteria levels remained around 3-4 $\log 10 \mathrm{cfu}$ with the powder, which was much lower than the bacterial concentrations observed with the capsule and sustained-release tablet (7-8 $\log 10 \mathrm{cfu}$ and up to $8.5 \log 10 \mathrm{cfu}$, respectively). Of note, the number of bacteria regularly increased over time in the ileal compartment for the sustained-release tablet, indicating progressive erosion of the tablet and associated release of the strain. From 180 to $240 \mathrm{~min}$ digestion, bacterial amounts were significantly higher with the sustained-release tablet compared to the powder $(P<0.001)$. At the end of digestion, the core of the tablet was still intact (data not shown) and contained $8.5 \log 10$ cfu. L. salivarius survival was also evaluated in the ileal effluents and in the gastrointestinal residue collected from all compartments at the end of digestion (data not shown). Global survival percentages were the lowest for the powder $(0.003 \pm 0.004 \%)$, followed by the capsule $(2.8 \pm 0.6 \%)(n=3)$. The highest survival was obtained for the sustained-release tablet $(17.0 \pm 1.8 \%, \mathrm{n}=3)$, combining free viable bacteria found in the digestive samples and the rest of the tablet at the end of digestion.
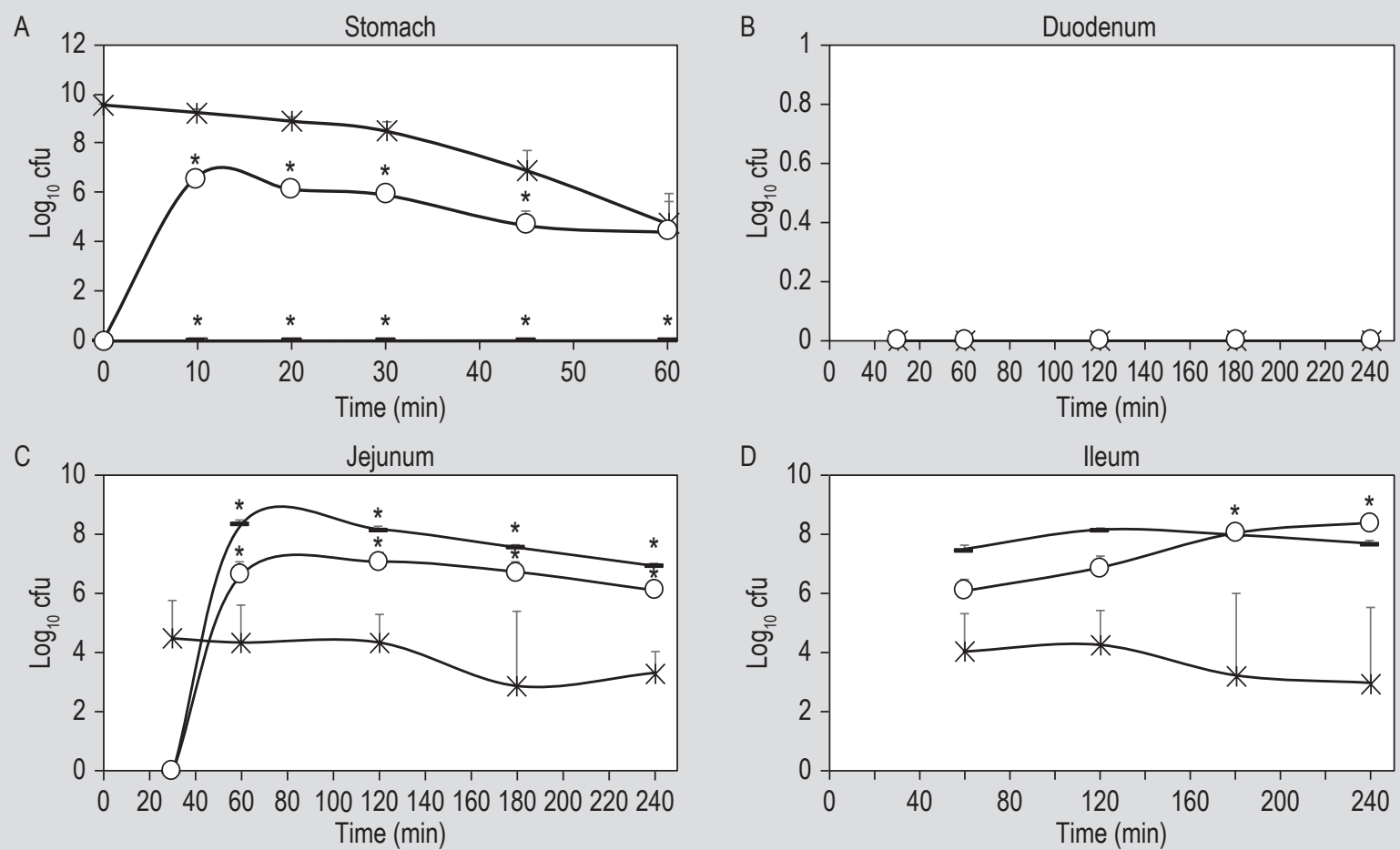

$$
\text { * Powder } \longrightarrow \text { Capsule }-\mathrm{O}-\mathrm{S}-\mathrm{r} \text { tablet }
$$

Figure 2. Impact of oral formulations on the survival of a Lactobacillus salivarius strain throughout the human simulated digestive tract. In order to assess the impact of three oral formulations, i.e. powder, HPMC capsule and sustained-release tablet (S-r tablet) on the viability of a $L$. salivarius strain during gastrointestinal transit, the bacterial suspension was introduced either into the TIM-1 stomach or in the ARCOL model from day 9 to day 15 after simplified static gastrointestinal digestion. Samples were regularly collected in each TIM-1 compartment during 4-hour in vitro digestion ((A) stomach, (B) duodenum, (C) jejunum and (D) ileum) or in the ARCOL throughout 21 day-fermentation assays ((E) control, (F) capsule, (G) S-r tablet) (during the stabilisation phase, probiotic treatment and post-treatment as described in Figure 1). Viability was assessed either by plate counts in the TIM-1 (no microbial background) or by PMAq-PCR in the ARCOL (resident colonic microbiota). Results are expressed as mean log10 cfu \pm standard deviation $(n=3)$ or median log10 gene copies [interquartile ranges] $(n=3)$. In the TIM-1 (A-D), only free cells were numerated by plating. Significant differences $(P<0.05)$ between capsule and tablet vs powder are indicated $\left(^{*}\right)$. In the ARCOL (E-G), significant differences $(P<0.05)$ were calculated between capsule or S-r tablet and control experiment $\left(^{*}\right)$. 

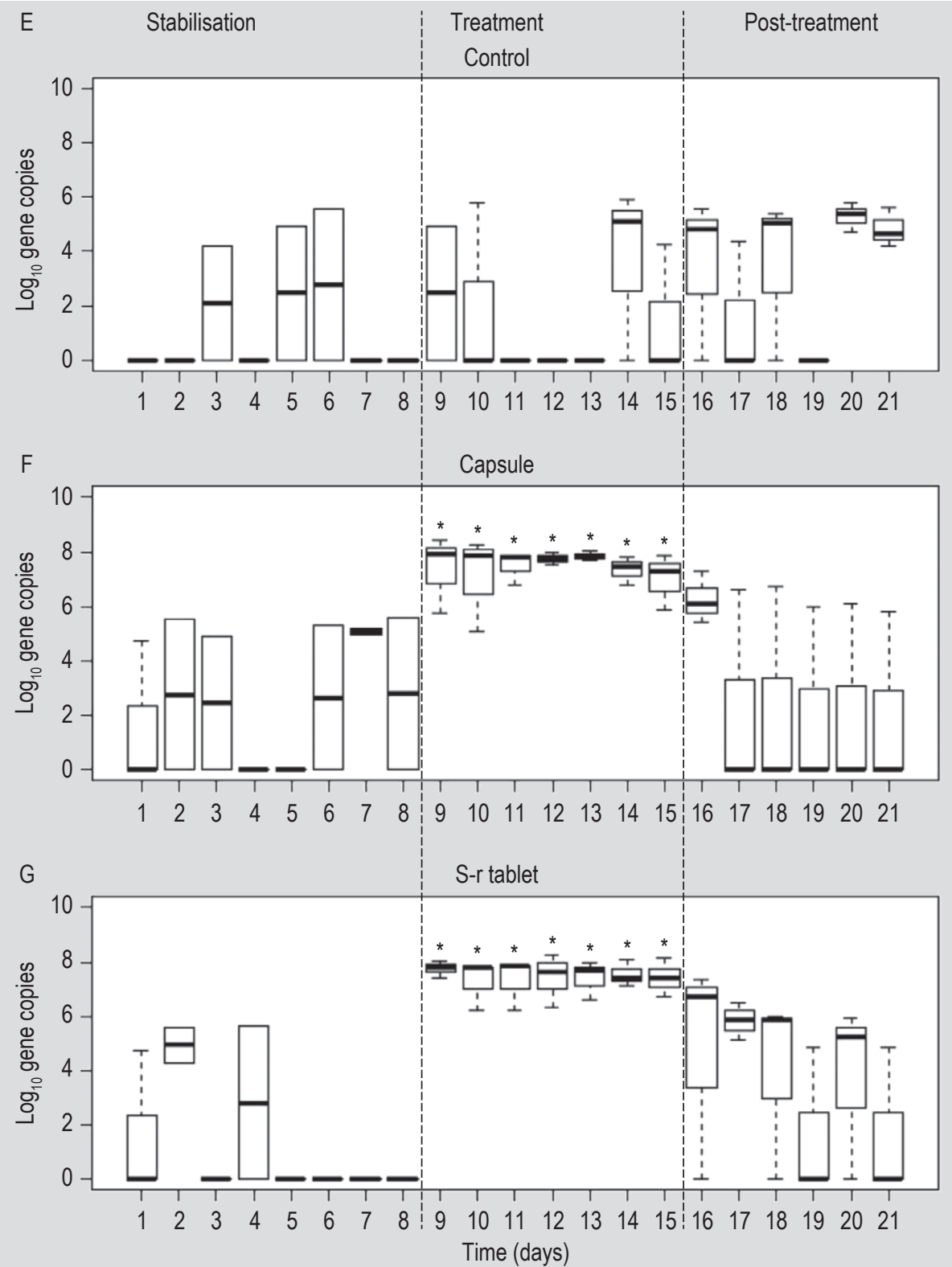

Figure 2. Continued.

\section{Colonic compartment}

Only the capsule and sustained-release forms, the most effective forms revealed in the TIM-1 experiments, were tested in the ARCOL system. In the control conditions (no probiotic treatment), basal $L$. salivarius levels ranging from 0 to $5 \log 10 \mathrm{cfu}$ were detected over the 21 day-fermentation in the bioreactor (Figure 2E). The highest levels corresponded to approximately $2 \log 10 \mathrm{cfu} / \mathrm{ml}$ (300 ml bioreactor volume) and could be assimilated to quantification of commensal strains. When the probiotic was added, bacterial numbers as determined by PMA-qPCR were significantly increased to around $8 \log 10 \mathrm{cfu}$ for the three donors $(P<0.05)$ (Figure 2F,G). This level was maintained during the 7 days of probiotic treatment and was similar between the two tested forms $(P>0.05)$. qPCR analysis revealed that during probiotic treatment $L$. salivarius was mostly alive, no difference being noticed between total qPCR and PMA-qPCR results (data not shown). After cessation of probiotic administration, L. salivarius concentrations rapidly decreased in the bioreactor for all donors to recover basal levels. Nevertheless, time to reach initial levels was widely donor-dependent, a longer persistence of the strain in the colon model being observed with donors 2 and 3 compared to donor 1 (data not shown). Of interest, on average, the initial level was reached on day 17 for the capsule compared to day 19 for the sustained-release tablet, suggesting that the latter form favours the persistence of the bacterial strain in the in vitro colonic medium. 


\section{Colonic microbiota composition and activity}

\section{Metabolic activity}

Redox potential of the fermentation medium stabilised around $-400 \mathrm{mV}$ in all bioreactors and this baseline value was not impacted by the probiotic treatment (data not shown). The total amount of gas produced over the 21 day fermentation was $5,608 \pm 4,244 \mathrm{ml}(\mathrm{n}=3)$ under control condition. This amount was higher with the probiotic treatment, reaching a total volume of gas of $8,266 \pm 687$ $\mathrm{ml}(\mathrm{n}=3)$ for capsule and even more for the sustainedrelease tablet $(9,381 \pm 2,503 \mathrm{ml}, \mathrm{n}=3$ ) (Figure 3). At the end of fermentations, the total volume of gas was significantly higher with the sustained-release tablet compared to control condition $(P<0.05)$, while no significant different was noticed at the end of the stabilisation phase. Gas composition fluctuated during stabilisation phase with a decrease in $\mathrm{N}_{2}$ (due to stop of initial bioreactor flushing) and $\mathrm{H}_{2}$ associated with an increase in $\mathrm{CO}_{2}$ relative percentages. Once stabilised, gas composition of the atmospheric phase under control condition was as follows: $1 \% \mathrm{H}_{2}, 4 \% \mathrm{CH}_{4}, 9 \% \mathrm{~N}_{2}, 83 \% \mathrm{CO}_{2}$, and $3 \% \mathrm{O}_{2} . \mathrm{O}_{2}$ levels remained during all the control fermentations below 1-3\%, indicating that anaerobiosis was efficiently maintained by the sole microbiota activity. Relative gas percentages were not modified by the probiotic whatever the tested form $(P>0.05)$. Major SCFAs, acetate, butyrate and propionate, were also measured daily during fermentations. At the end of the stabilisation phase, total SCFA concentration in the control bioreactors was $123 \pm 19 \mathrm{mM}$ with $55 \%$ acetate, $16 \%$ butyrate and $29 \%$ propionate (Figure 4 ). Total SCFA concentrations as well as relative percentages of acetate, butyrate and propionate were not significantly modified by probiotic administration, whatever the galenic form $(P>0.05)$. Similarly, concentrations of the three main SCFAs were not altered by the probiotic, with the exception of propionate which was significantly decreased compared to control condition with both the capsule and sustainedrelease tablet, but only at day 11 (data not shown).

\section{Microbiota composition}

Microbiota composition was determined at the phylum (Supplementary Figure S1) and family levels (Figure 5A). During control experiments, microbiota stabilised in the bioreactors with clear donor-dependent variability (Supplementary Figure S2). In accordance with in vivo data in humans, the main phyla detected were Bacteroidetes, Firmicutes and Proteobacteria, and major families were Bacteroidaceae, Burkholderiaceae, Ruminococcaceae, Rikenellaceae and Lachnospiraceae. Probiotic treatment led to changes in gut microbiota composition. Although modifications were donor-dependent, they were mainly characterised by a decrease in Bacteroidaceae (27\% abundance under control conditions vs 14 and $9 \%$ with the probiotic administered within capsule and sustainedrelease tablet, respectively).together with an increase in Rikenellaceae and Ruminococcaceae. Microbial profiles did not differ between the two galenic formulations. Lastly, post-treatment phase was associated with microbial profiles which remained close to those observed during probiotic administration, even if relative microbial abundances clearly differed at some time points (e.g. day 21 for the capsule with a high Veillonellaceae abundance). Alpha diversity indexes showed that neither the day of fermentation (data not shown) nor the treatment (control vs capsule or tablet) had an influence on microbial diversity $(P>0.05)$ (Figure $5 \mathrm{~B})$. Multidimentional scaling (MDS) plots using the Bray-Curtis dissimilarity index confirmed the absence of formulation effect on microbiota composition (Supplementary Figure S2).

\section{Discussion}

L. salivarius strains have been already used as probiotics in humans to prevent or treat a variety of chronic diseases including asthma and atopic dermatitis and to a lesser extent bacterial or fungal infections (Chaves et al., 2017). In this study, we showed the value of a sustained-release tablet in delaying the release of a $L$. salivarius strain in the gut, and increasing its survival in the human simulated stomach and small intestine, compared to more classical formulations such as powder and HPMC capsule.

During gastrointestinal passage, probiotics face two major stresses, acidic $\mathrm{pH}$ in the stomach and bile salts in the small intestine. Even if the effect are obviously strain-dependent, L. salivarius strains were shown to be sensitive to low $\mathrm{pH}$, like many other lactic acid bacteria (Fuochi et al., 2015; Sanhueza et al., 2015). In order to increase probiotic survival during transit through the human stomach, various gastro-resistant formulations have been developed for Lactobacillus spp. strains, using various matrices mainly based on alginate (Atia et al., 2018; Cheow et al., 2014; Ding and Shah, 2009; Villena et al., 2015) and ovalbumin (Govender et al., 2015). In the present study, we showed for the first time that HPMC capsule effectively protected the $L$. salivarius strain tested against acid $\mathrm{pH}$ as no bacteria was released in the TIM stomach and subsequent survival was improved in the distal parts of the small intestine. Hydroxypropylmethylcellulose is highly resistant to low $\mathrm{pH}$ (up to 1-2) but disintegrates when $\mathrm{pH}$ increases to higher values such as those found in the human small intestine (Cole et al., 2002). Such material allows the targeted delivery of probiotics but also of other acid-sensitive compounds, such as enzymes to the intestine (Marzorati et al., 2015).

Once the gastric barrier crossed, probiotics have to deal with bile salts, produced by the liver and delivered into the duodenum. Bile is a well-known bactericidal agent targeting and disrupting bacterial cell membrane (Begley et 


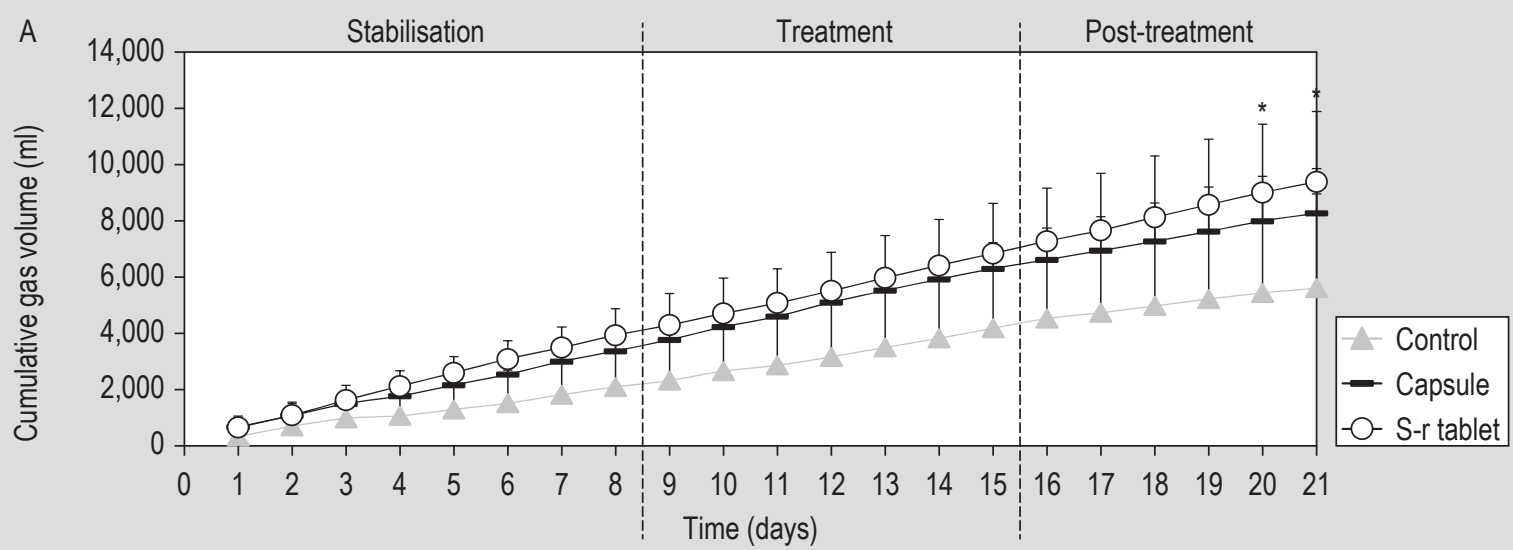

B

Stabilisation

Treatment

Post-treatment
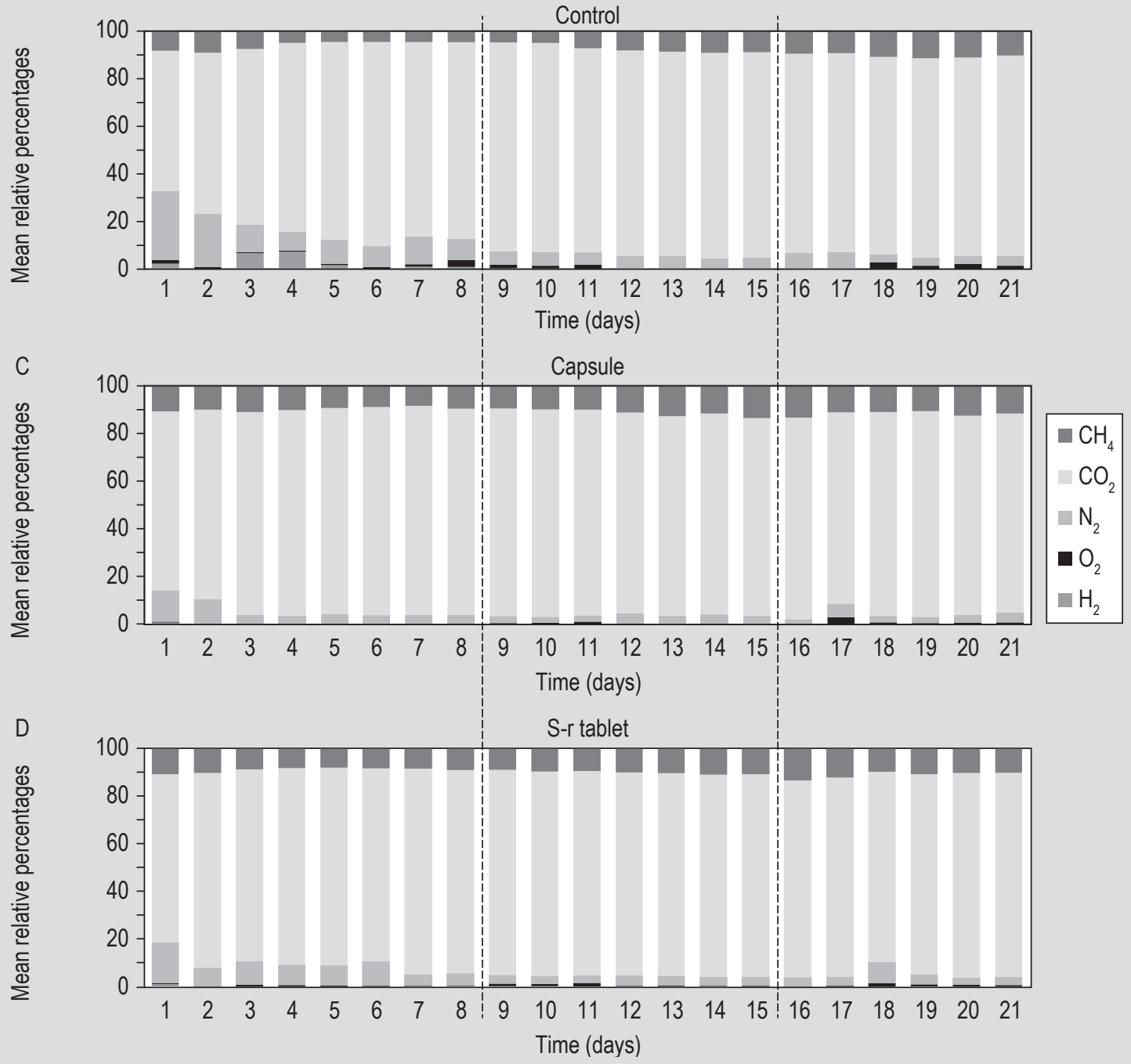

Figure 3. Impact of oral formulations of a Lactobacillus salivarius strain on gas production in the ARCOL model. Three ARCOL bioreactors were inoculated with the same faecal suspension and ran in parallel for 21 days. Experiments were performed in triplicate with the faeces from three different healthy adult donors. After 8 days of stabilisation, the capsule or sustained-release tablet (S-r tablet) containing the $L$. salivarius strain was administered once a day for 7 days. Control bioreactors received no probiotic treatment. Three periods were determined as follows: stabilisation (day 0 to 8), probiotic treatment (day 9 to 15) and post-treatment phases (day 16 to 21). Samples were collected daily from the atmospheric phase of bioreactors to determine (A) total gas production (in $\mathrm{ml}$ ) and gas composition by gas chromatography (in relative percentages, per treatment: (B) control, (C) capsule, (D) S-r tablet). Results are expressed as means \pm standard deviation $(n=3)$. 


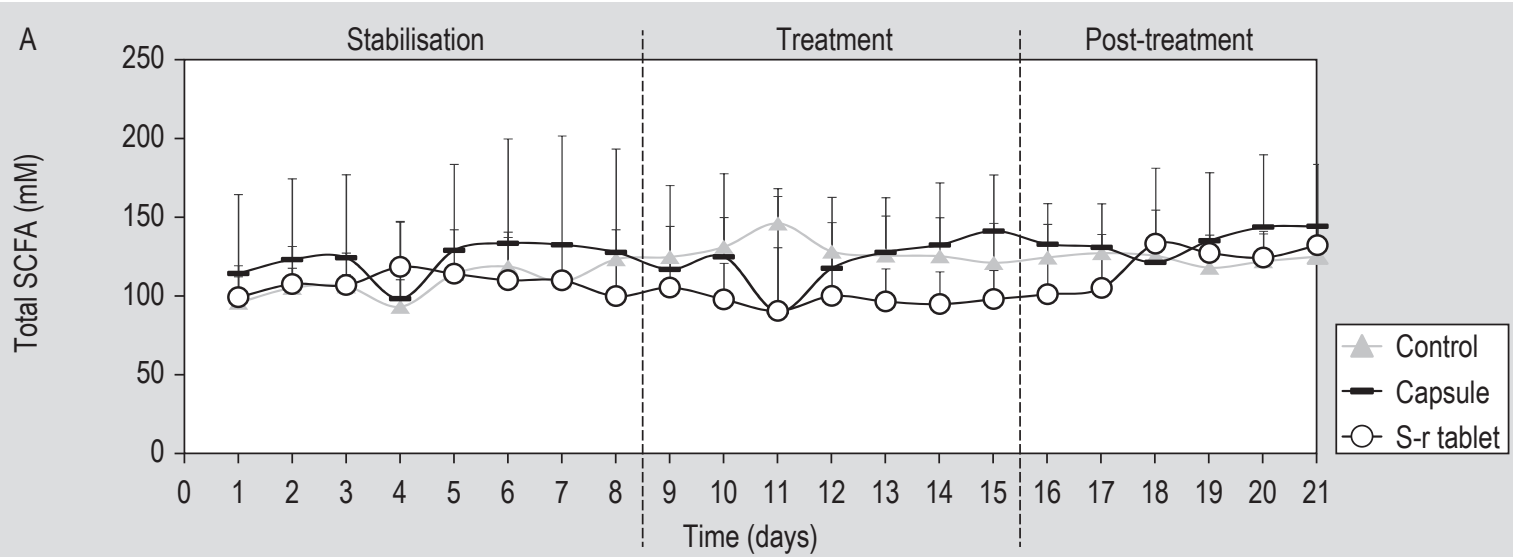

B

Stabilisation

Treatment

Post-treatment

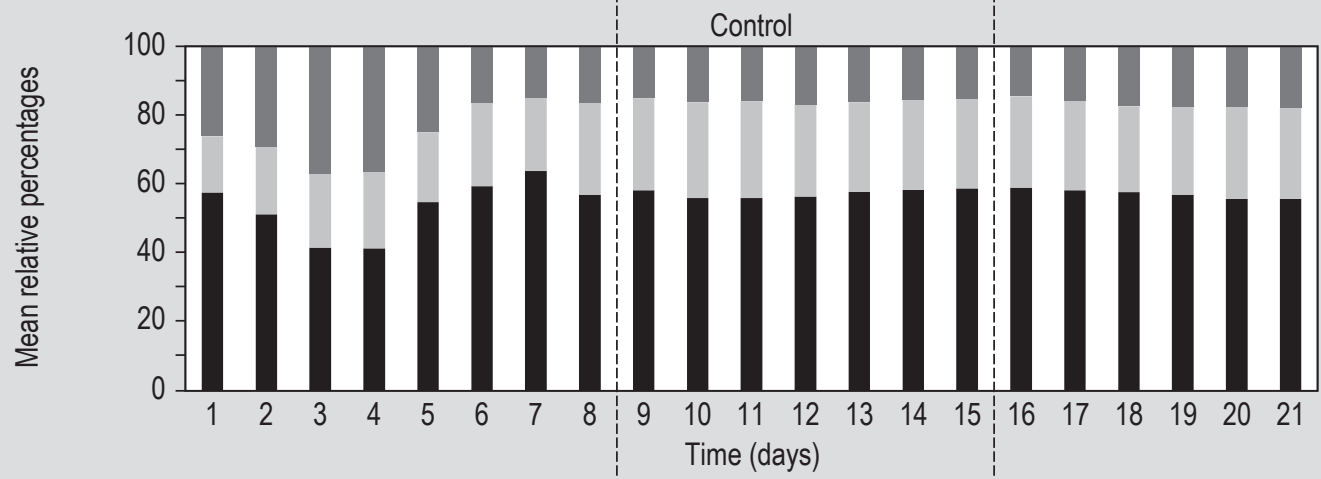

C

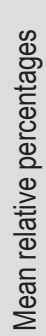

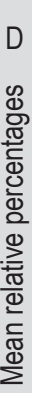

Time (days)

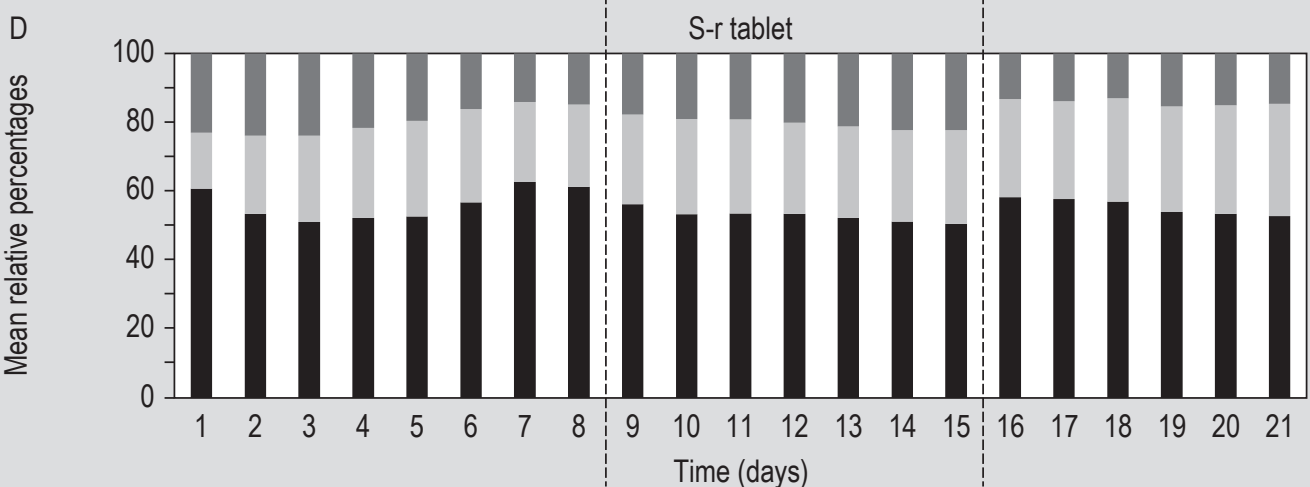

Figure 4. Impact of oral formulations of a Lactobacillus salivarius strain on short chain fatty acid production in the ARCOL model. Experiments were performed as described in Figure 3. Samples were collected daily from bioreactor fermentation medium. Main SCFAs (acetate, propionate and butyrate) were analysed by high performance liquid chromatography. Results are either expressed as (A) mean total SCFA concentrations \pm standard deviation (in mM) or mean relative percentages per treatment ((B) control, (C) capsule, (D) S-r tablet). Significant differences $(P<0.05)$ between capsule and tablet versus control experiment are indicated $\left({ }^{*}\right)$. 


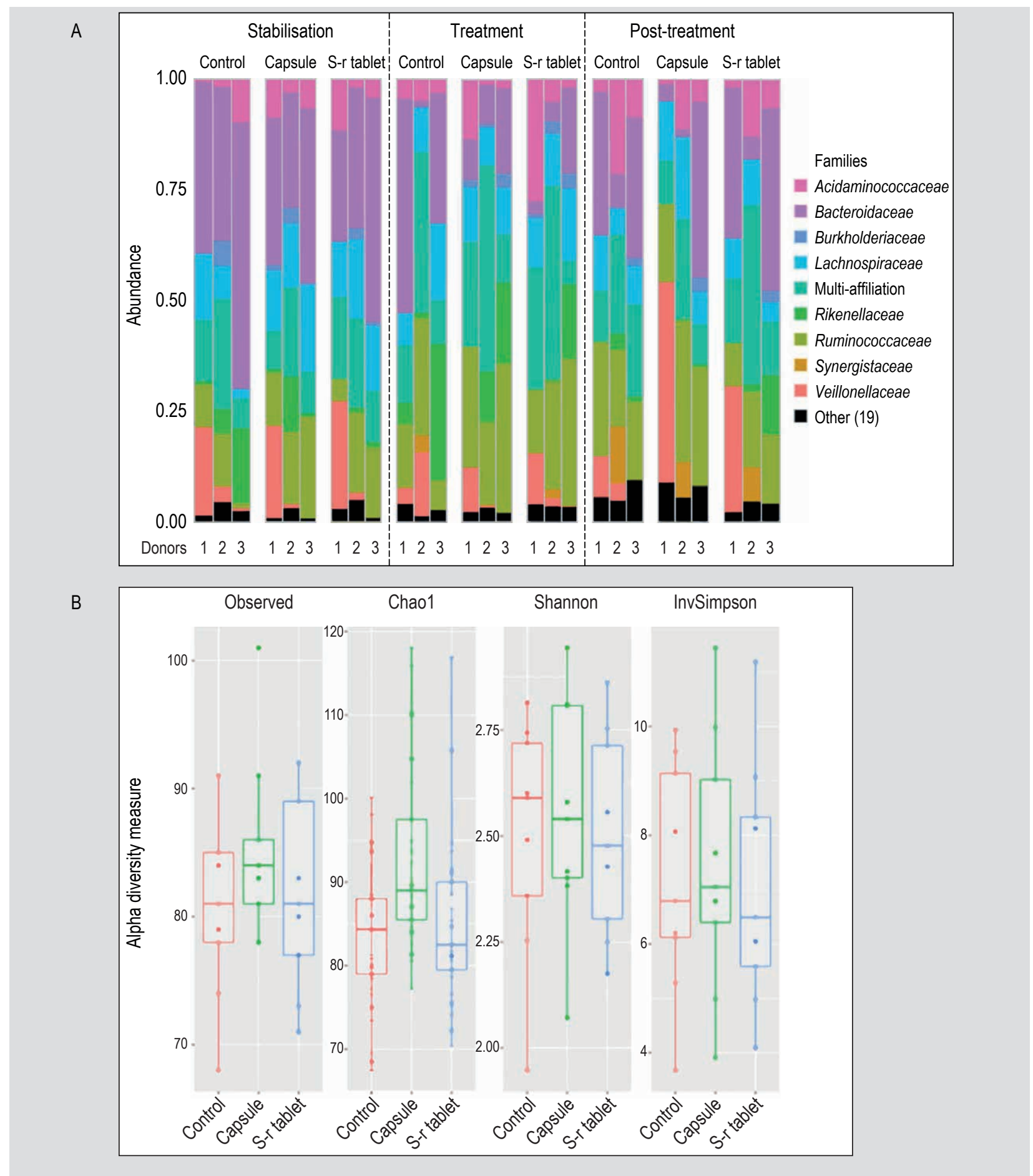

Figure 5. Impact of oral formulations of a Lactobacillus salivarius strain on gut microbiota composition at the family level and alpha-diversity indexes. Experiments were performed as described in Figure 3. Samples were collected daily from bioreactor fermentation medium. For each of the three donors, (A) microbial composition was determined at the family level by $16 \mathrm{~S}$ rRNA gene sequencing and expressed as relative abundances. (B) Alpha-diversity index was determined at OTU level.

al., 2006). In Lactobacillus spp., bile resistance mechanisms include efflux systems and bile salt hydrolyse enzymes (Fang et al., 2009; Lv et al., 2017; Wang et al., 2015). The L. salivarius strain tested was shown to be highly sensitive to high concentrations of bile salts as no viable bacteria was recovered in the duodenum whatever the galenic formulation. Bile salt hydrolase enzymatic activity was not tested in the strain but analysis of DNA sequence revealed the presence of bsh 1 but not bsh2, 3 or 4 (Barache et al., 2020), as previously described for other $L$. salivarius strains 
(Fang et al., 2009; Wang et al., 2012). Like for acid sensitivity, bile tolerance is widely strain-dependent (Fang et al., 2009). The high sensitivity of the strain of $L$. salivarius emphasises the interest of using not only gastro-resistant forms but rather sustained-release formulations that can protect the probiotic from both acid $\mathrm{pH}$ and bile salts by releasing bacteria only in the distal parts of the small intestine or later in the colon.

Different sustained-release formulations have been developed for Lactobacillus spp, based on hypromellose (other name for hydroxypropylmethylcellulose) (Marzorati et al., 2015) and alginate/inulin (Atia et al., 2018). The sustained-release tablet we tested contained hydroxypropylmethylcellulose and different excipients for direct compression. The degree of substitution and powder properties (i.e. particle size) of hydroxypropylmethylcellulose were controlled to be suitable for sustained release preparations. Compared to a classical powder form, the sustained-release tablet allowed an increase in L. salivarius survival rate in the TIM-1 ileal effluents of $4 \log$ higher. Release of the L. salivarius strain was delayed as bacteria were mostly recovered in the jejunal end ileal compartments. Bacterial concentrations of 8-9 logs were found, which is much higher that the minimal dose required for a probiotic effect, stated at $10^{6} \mathrm{cfu} / \mathrm{ml}$ in the small intestine (Minelli and Benini, 2008). Moreover, at the end of digestion, the core of the tablet found intact in the ileal effluents contained more than $8 \log$ bacteria. Therefore, all of these bacteria would reach the colon in a viable state, which is necessary for probiotic activity when this site is targeted for action.

Once in the colon, probiotic strains have to compete with resident microbiota for nutrients and ecological niches (Pereira and Berry, 2017), but are less subjected to physicochemical stresses than in the upper gut. In the present study, repeated administration of the probiotic (daily administration) was required to maintain a high colonisation level in the ARCOL system (8 logs). Similar observation was made by Klingberg and Budde (2006) who found viable $L$. salivarius in the faecal human samples during probiotic administration and none six days after the end of treatment. The sustained-release tablet tended to maintain higher concentrations of $L$. salivarius compared to HPMC capsule two days after cessation of administration. To our knowledge, this study is the first one that assessed the effect of galenic formulations on the release, survival and interactions with gut microbiota of a L. salivarius strain in simulated human colonic conditions.

Whatever the tested formulation (HPMC capsule and sustained-release tablet), L. salivarius induced a decrease in Bacteroidaceae associated with an increase in Rikenellaceae and Ruminococcaceae. Bacteroidaceae are involved in almost all bacterial functional gene categories in the human gut microbiota and the effect of Lactobacillus strains on this population is highly study-dependent, making difficult any conclusion on a good or bad effect of the probiotic strain (Wexler, 2007; Wexler and Goodman, 2017). On the contrary, as Ruminococcus species are consistently present in the healthy human gut, this suggests a possible role in maintaining a healthy state (Park, 2018). Even if some of the modifications were common to the three donors, they remained donor-dependent, which suggests that the probiotic may not have the same effects in each individual. In accordance with this statement, previous studies on other Lactobacillus strains have evidenced that probiotic effect was closely related to the composition of individual's basal gut microbiota (Hou et al., 2020). Up to now, the few studies that have investigated the effect of L. salivarius on human intestinal microbiota (Drago et al., 2012; Larsen et al., 2013) have been performed on faecal (and not on colonic) samples and have followed only specific bacterial populations, making difficult any comparison with our study. Of note, a very recent study performed by Yang et al. (2020) showed that a mix of L. salivarius and L. reuteri strains induced both a decrease in Bacteroidetes and an increase in Ruminococcaceae relative abundances in piglet caecal microbiota, which is in line with our results. By using mutants, Riboulet-Bisson et al. (2012) showed that the effect of L. salivarius on gut microbiota seems to be, at least partially, bacteriocin-dependant. Lastly, the modifications of gut microbiota induced by L. salivarius were not associated with major changes in microbial activity as assessed by measurement of gas and SCFA. These results are in accordance with those of Larsen et al. (2013) who administered a L. salivarius strain to obese adolescents for a 12-week period.

\section{Conclusions}

This study showed the interest of an HPMC capsule to increase the survival of a L. salivarius strain during gastric passage and showed the ability of a sustained-release tablet to increase the global viability of the probiotic tested in the human intestine and thus target the colon compartment. These results were obtained by combining two complementary in vitro gut models, the TIM-1 and ARCOL systems, confirming their potential as a relevant alternative to in vivo assays in preclinical phases for the assessment of various probiotic formulations. As the impact of galenic forms is strain-dependent, such in vitro approach could be applied to the testing of many other probiotic strains. Furthermore, the flexibility of in vitro gut models makes it easy to imagine extending their use for probiotic formulation assessment to different food matrices (Cordonnier et al., 2015; Ribnicky et al., 2014; Uriot et al., 2016), various age groups, such as infants or elderly subjects (Denis et al., 2016; Roussel et al., 2016), and pathological conditions such as obesity (Bussolo de Souza et al., 2014). 


\section{Supplementary material}

Supplementary material can be found online at https://doi. org/10.3920/BM2020.0187.

Table S1. Parameters of in vitro gut models used to reproduce digestive conditions of a healthy human adult.

Figure S1. Impact of oral formulation of a Lactobacillus salivarius strain on gut microbiota composition at the phylum level.

Figure S2. Multidimensional scaling of gut microbiota composition.

\section{Acknowledgements}

We are grateful to the genotoul bioinformatics platform Toulouse Midi-Pyrénées and Sigenae group for providing help and storage resources thanks to Galaxy instance http:// sigenae-workbench.toulouse.inra.fr. This study was funding by a grant from Auvergne Region and Larena Santé.

\section{References}

Atia, A., Gomaa, A., Fernandez, B., Subirade, M. and Fliss, I., 2018. Study and understanding behavior of alginate-inulin synbiotics beads for protection and delivery of antimicrobial-producing probiotics in colonic simulated conditions. Probiotics Antimicrobial Proteins 10: 157-167. https://doi.org/10.1007/s12602-017-9355-x

Azad, M.A.K., Sarker, M., Li, T. and Yin, J., 2018. Probiotic species in the modulation of gut microbiota: an overview. Biomed Research International 2018: 9478630. https://doi.org/10.1155/2018/9478630

Barache, N., Ladjouzi, R., Belguesmia, Y., Bendali, F. and Drider, D., 2020. Abundance of Lactobacillus plantarum strains with beneficial attributes in blackberries (Rubus sp.), fresh figs (Ficus carica), and prickly pears (Opuntia ficus-indica) grown and harvested in Algeria. Probiotics Antimicrobial Proteins 12: 1514-1523. https:// doi.org/10.1007/s12602-020-09632-z

Begley, M., Hill, C. and Gahan, C.G.M., 2006. Bile salt hydrolase activity in probiotics. Applied and Environmental Microbiology 72: 1729-1738. https://doi.org/10.1128/AEM.72.3.1729-1738.2006

Blanquet-Diot, S., Denis, S., Chalancon, S., Chaira, F., Cardot, J.-M., Alric, M., 2012. Use of artificial digestive systems to investigate the biopharmaceutical factors influencing the survival of probiotic yeast during gastrointestinal transit in humans. Pharmaceutical Research 29: 1444-1453. https://doi.org/10.1007/s11095-011-0620-5

Bussolo de Souza, C., Roeselers, G., Troost, F., Jonkers, D., Koenen, M.E. and Venema, K., 2014. Prebiotic effects of cassava bagasse in TNO's in vitro model of the colon in lean versus obese microbiota. Journal of Functional Foods 11: 210-220. https://doi.org/10.1016/j. jff.2014.09.019

Campana, R., Van Hemert, S. and Baffone, W., 2017. Strain-specific probiotic properties of lactic acid bacteria and their interference with human intestinal pathogens invasion. Gut Pathogens 9: 12. https://doi.org/10.1186/s13099-017-0162-4
Chaves, B.D., Brashears, M.M. and Nightingale, K.K., 2017. Applications and safety considerations of Lactobacillus salivarius as a probiotic in animal and human health. Journal of Applied Microbiology 123: 18-28. https://doi.org/10.1111/jam.13438

Cheow, W.S., Kiew, T.Y. and Hadinoto, K., 2014. Controlled release of Lactobacillus rhamnosus biofilm probiotics from alginate-locust bean gum microcapsules. Carbohydrate Polymers 103: 587-595. https://doi.org/10.1016/j.carbpol.2014.01.036

Cole, E.T., Scott, R.A., Connor, A.L., Wilding, I.R., Petereit, H.U., Schminke, C., Beckert, T. and Cadé, D., 2002. Enteric coated HPMC capsules designed to achieve intestinal targeting. International Journal of Pharmaceutics 231: 83-95. https://doi.org/10.1016/s03785173(01)00871-7

Cordonnier, C., Thévenot, J., Etienne-Mesmin, L., Denis, S., Alric, M., Livrelli, V. and Blanquet-Diot, S., 2015. Dynamic in vitro models of the human gastrointestinal tract as relevant tools to assess the survival of probiotic strains and their interactions with gut microbiota. Microorganisms 3: 725-745. https://doi.org/10.3390/ microorganisms 3040725

Denis, S., Sayd, T., Georges, A., Chambon, C., Chalancon, S., SantéLhoutellier, V. and Blanquet-Diot, S., 2016. Digestion of cooked meat proteins is slightly affected by age as assessed using the dynamic gastrointestinal TIM model and mass spectrometry. Food Functions 7: 2682-2691. https://doi.org/10.1039/c6fo00120c

Ding, W.K. and Shah, N.P., 2009. An improved method of microencapsulation of probiotic bacteria for their stability in acidic and bile conditions during storage. Journal of Food Science 74: M53-61. https://doi.org/10.1111/j.1750-3841.2008.01030.x

Drago, L., Toscano, M., De Vecchi, E., Piconi, S. and Iemoli, E., 2012. Changing of fecal flora and clinical effect of $L$. salivarius LS01 in adults with atopic dermatitis. Journal of Clinical Gastroenterology 46, Suppl.: S56-63. https://doi.org/10.1097/MCG.0b013e318265ef38

Eiberger, I., Bley, H., Molimard, P., Maathuis, A. and Venema, K., 2011. Evaluation of the appropriate galenical technology for the site specific delivery of probiotic bacteria. In: Proceedings of Vitagora. Vitagora, Dijon, France, p. 7.

Fang, F., Li, Y., Bumann, M., Raftis, E.J., Casey, P.G., Cooney, J.C., Walsh, M.A. and O'Toole, P.W., 2009. Allelic variation of bile salt hydrolase genes in Lactobacillus salivarius does not determine bile resistance levels. Journal of Bacteriology 191: 5743-5757. https:// doi.org/10.1128/JB.00506-09

Food and Agricultural Organization of the United Nations and World Health Organization (FAO/WHO), 2002. Joint FAO/WHO working group report on drafting guidelines for the evaluation of probiotics in food. FAO/WHO, Rome/Geneva, Italy/Switzerland.

Fuochi, V., Petronio, G.P., Lissandrello, E., and Furneri, P.M., 2015. Evaluation of resistance to low $\mathrm{pH}$ and bile salts of human Lactobacillus spp. isolates. International Journal of Immunopathology and Pharmacology 28: 426-433. https://doi.org/10.1177/ 0394632015590948

Gately, N.M. and Kennedy, J.E., 2017. The development of a meltextruded shellac carrier for the targeted delivery of probiotics to the colon. Pharmaceutics 9: 38. https://doi.org/10.3390/ pharmaceutics 9040038 
Govender, M., Choonara, Y.E., Van Vuuren, S., Kumar, P., Du Toit, L.C. and Pillay, V., 2015. A gastro-resistant ovalbumin bi-layered minitablet-in-tablet system for the delivery of Lactobacillus acidophilus probiotic to simulated human intestinal and colon conditions. Journal of Pharmacy and Pharmacology 67: 939-950. https://doi. org/10.1111/jphp.12389

He, M. and Shi, B., 2017. Gut microbiota as a potential target of metabolic syndrome: the role of probiotics and prebiotics. Cell and Bioscience 7: 54. https://doi.org/10.1186/s13578-017-0183-1

Hill, C., Guarner, F., Reid, G., Gibson, G.R., Merenstein, D.J., Pot, B., Morelli, L., Canani, R.B., Flint, H.J., Salminen, S., Calder, P.C. and Sanders, M.E., 2014. Expert consensus document. The International Scientific Association for Probiotics and Prebiotics consensus statement on the scope and appropriate use of the term probiotic. Nature Reviews Gastroenterology \& Hepatology 11: 506-514. https:// doi.org/10.1038/nrgastro.2014.66

Holowacz, S., Blondeau, C., Guinobert, I., Guilbot, A., Hidalgo, S. and Bisson, J.F., 2018. Lactobacillus salivarius LA307 and Lactobacillus rhamnosus LA305 attenuate skin inflammation in mice. Beneficial Microbes 9: 299-309. https://doi.org/10.3920/BM2017.0084

Hou, Q., Zhao, F., Liu, W., Lv, R., Khine, W.W.T., Han, J., Sun, Z., Lee, Y.-K. and Zhang, H., 2020. Probiotic-directed modulation of gut microbiota is basal microbiome dependent. Gut Microbes 12: 1736974. https://doi.org/10.1080/19490976.2020.1736974

Klingberg, T.D. and Budde, B.B., 2006. The survival and persistence in the human gastrointestinal tract of five potential probiotic lactobacilli consumed as freeze-dried cultures or as probiotic sausage. International Journal of Food Microbiology 109: 157-159. https://doi.org/10.1016/j.ijfoodmicro.2006.01.014

Larsen, N., Vogensen, F.K., Gøbel, R.J., Michaelsen, K.F., Forssten, S.D., Lahtinen, S.J., Jakobsen, M., 2013. Effect of Lactobacillus salivarius Ls-33 on fecal microbiota in obese adolescents. Clinical Nutrition 32: 935-940. https://doi.org/10.1016/j.clnu.2013.02.007

Lv, L.-X., Yan, R., Shi, H.-Y., Shi, D., Fang, D.-Q., Jiang, H.-Y., Wu, W.-R., Guo, F.-F., Jiang, X.-W., Gu, S.-L., Chen, Y.-B., Yao, J. and Li, L.-J., 2017. Integrated transcriptomic and proteomic analysis of the bile stress response in probiotic Lactobacillus salivarius LI01. Journal of Proteomics 150: 216-229. https://doi.org/10.1016/j.jprot.2016.08.021

Marteau, P., Minekus, M., Havenaar, R. and Huis in't Veld, J.H., 1997. Survival of lactic acid bacteria in a dynamic model of the stomach and small intestine: validation and the effects of bile. Journal of Dairy Science 80: 1031-1037. https://doi.org/10.3168/jds.S00220302(97)76027-2

Marzorati, M., Possemiers, S., Verhelst, A., Cadé, D., Madit, N. and Van de Wiele, T., 2015. A novel hypromellose capsule, with acid resistance properties, permits the targeted delivery of acid-sensitive products to the intestine. LWT - Food Science and Technology 60: 544-551. https://doi.org/10.1016/j.lwt.2014.08.040

Messaoudi, S., Manai, M., Kergourlay, G., Prévost, H., Connil, N., Chobert, J.-M., Dousset, X., 2013. Lactobacillus salivarius: bacteriocin and probiotic activity. Food Microbiology 36: 296-304. https://doi.org/10.1016/j.fm.2013.05.010

Minelli, E.B. and Benini, A., 2008. Relationship between number of bacteria and their probiotic effects. Microbial Ecology in Health and Disease 20: 180-183. https://doi.org/10.1080/08910600802408095
Park, W., 2018. Gut microbiomes and their metabolites shape human and animal health. Journal of Microbiology 56: 151-153. https:// doi.org/10.1007/s12275-018-0577-8

Patrignani, F., Siroli, L., Parolin, C., Serrazanetti, D.I., Vitali, B. and Lanciotti, R., 2019. Use of Lactobacillus crispatus to produce a probiotic cheese as potential gender food for preventing gynaecological infections. PLoS ONE 14: e0208906. https://doi. org/10.1371/journal.pone.0208906

Pereira, F.C. and Berry, D., 2017. Microbial nutrient niches in the gut. Environmental Microbiology 19: 1366-1378. https://doi. org/10.1111/1462-2920.13659

Plaza-Diaz, J., Ruiz-Ojeda, F.J., Gil-Campos, M. and Gil, A., 2019. Mechanisms of action of probiotics. Advanced Nutrition 10: S49-S66. https://doi.org/10.1093/advances/nmy063

Ribnicky, D.M., Roopchand, D.E., Oren, A., Grace, M., Poulev, A., Lila, M.A., Havenaar, R. and Raskin, I., 2014. Effects of a high fat meal matrix and protein complexation on the bioaccessibility of blueberry anthocyanins using the TNO gastrointestinal model (TIM-1). Food Chemistry 142: 349-357. https://doi.org/10.1016/j. foodchem.2013.07.073

Riboulet-Bisson, E., Sturme, M.H.J., Jeffery, I.B., O’Donnell, M.M., Neville, B.A., Forde, B.M., Claesson, M.J., Harris, H., Gardiner, G.E., Casey, P.G., Lawlor, P.G., O’Toole, P.W. and Ross, R.P., 2012. Effect of Lactobacillus salivarius bacteriocin Abp118 on the mouse and pig intestinal microbiota. PLoS ONE 7: e31113. https://doi. org/10.1371/journal.pone.0031113

Roussel, C., Cordonnier, C., Galia, W., Le Goff, O., Thévenot, J., Chalancon, S., Alric, M., Thevenot-Sergentet, D., Leriche, F., Van de Wiele, T., Livrelli, V. and Blanquet-Diot, S., 2016. Increased EHEC survival and virulence gene expression indicate an enhanced pathogenicity upon simulated pediatric gastrointestinal conditions. Pediatric Research 80: 734-743. https://doi.org/10.1038/pr.2016.144 Sanhueza, E., Paredes-Osses, E., González, C.L. and García, A., 2015. Effect of $\mathrm{pH}$ in the survival of Lactobacillus salivarius strain UCO_979C wild type and the $\mathrm{pH}$ acid acclimated variant. Electronic Journal of Biotechnology 18: 343-346. https://doi.org/10.1016/j. ejbt.2015.06.005

Sniffen, J.C., McFarland, L.V., Evans, C.T. and Goldstein, E.J.C., 2018. Choosing an appropriate probiotic product for your patient: an evidence-based practical guide. PLoS ONE 13: e0209205. https:// doi.org/10.1371/journal.pone.0209205

Soares, M.B., Martinez, R.C.R., Pereira, E.P.R., Balthazar, C.F., Cruz, A.G., Ranadheera, C.S. and Sant'Ana, A.S., 2019. The resistance of Bacillus, Bifidobacterium, and Lactobacillus strains with claimed probiotic properties in different food matrices exposed to simulated gastrointestinal tract conditions. Food Research International 125: 108542. https://doi.org/10.1016/j.foodres.2019.108542

Surono, I., Verhoeven, J., Verbruggen, S. and Venema, K., 2018. Microencapsulation increases survival of the probiotic Lactobacillus plantarum IS-10506, but not Enterococcus faecium IS-27526 in a dynamic, computer-controlled in vitro model of the upper gastrointestinal tract. Journal of Applied Microbiology 124: 16041609. https://doi.org/10.1111/jam.13740 
Thévenot, J., Cordonnier, C., Rougeron, A., Le Goff, O., Nguyen, H.T.T., Denis, S., Alric, M., Livrelli, V. and Blanquet-Diot, S., 2015. Enterohemorrhagic Escherichia coli infection has donor-dependent effect on human gut microbiota and may be antagonized by probiotic yeast during interaction with Peyer's patches. Applied Microbiology and Biotechnology 99: 9097-9110. https://doi.org/10.1007/s00253015-6704-0

Uriot, O., Galia, W., Awussi, A.A., Perrin, C., Denis, S., Chalancon, S., Lorson, E., Poirson, C., Junjua, M., Le Roux, Y., Alric, M., Dary, A., Blanquet-Diot, S. and Roussel, Y., 2016. Use of the dynamic gastro-intestinal model TIM to explore the survival of the yogurt bacterium Streptococcus thermophilus and the metabolic activities induced in the simulated human gut. Food Microbiology 53: 18-29. https://doi.org/10.1016/j.fm.2015.05.007

Van den Abbeele, P., Roos, S., Eeckhaut, V., MacKenzie, D.A., Derde, M., Verstraete, W., Marzorati, M., Possemiers, S., Vanhoecke, B., Van Immerseel, F. and Van de Wiele, T., 2012. Incorporating a mucosal environment in a dynamic gut model results in a more representative colonization by lactobacilli. Microbial Biotechnology 5: 106-115. https://doi.org/10.1111/j.1751-7915.2011.00308.x

Venema, K., Verhoeven, J., Beckman, C. and Keller, D., 2020. Survival of a probiotic-containing product using capsule-within-capsule technology in an in vitro model of the stomach and small intestine (TIM-1). Beneficial Microbes 11: 403-409. https://doi.org/10.3920/ BM2019.0209

Venema, K., Verhoeven, J., Verbruggen, S., Espinosa, L. and Courau, S., 2019. Probiotic survival during a multi-layered tablet development as tested in a dynamic, computer-controlled in vitro model of the stomach and small intestine (TIM-1). Letters in Applied Microbiology 69: 325-332. https://doi.org/10.1111/lam.13211
Vijaya Kumar, B., Vijayendra, S.V.N. and Reddy, O.V.S., 2015. Trends in dairy and non-dairy probiotic products - a review. Journal of Food Science and Technology 52: 6112-6124. https://doi.org/10.1007/ s13197-015-1795-2

Villena, M.J.M., Lara-Villoslada, F., Martínez, M.A.R. and Hernández, M.E.M., 2015. Development of gastro-resistant tablets for the protection and intestinal delivery of Lactobacillus fermentum CECT 5716. International Journal of Pharmaceutics 487: 314-319. https:// doi.org/10.1016/j.ijpharm.2015.03.078

Wang, G., Li, D., Ma, X., An, H., Zhai, Z., Ren, F. and Hao, Y., 2015. Functional role of oppA encoding an oligopeptide-binding protein from Lactobacillus salivarius Ren in bile tolerance. Journal of Industrial Microbiology and Biotechnology 42: 1167-1174. https:// doi.org/10.1007/s10295-015-1634-5

Wang, Z., Zeng, X., Mo, Y., Smith, K., Guo, Y. and Lin, J., 2012. Identification and characterization of a bile salt hydrolase from Lactobacillus salivarius for development of novel alternatives to antibiotic growth promoters. Applied and Environmental Microbiology 78: 8795-8802. https://doi.org/10.1128/AEM.02519-12 Wexler, A.G. and Goodman, A.L., 2017. An insider's perspective: Bacteroides as a window into the microbiome. Nature Microbiology 2: 17026. https://doi.org/10.1038/nmicrobiol.2017.26

Wexler, H.M., 2007. Bacteroides: the good, the bad, and the nittygritty. Clinical Microbiology Reviews 20: 593-621. https://doi. org/10.1128/CMR.00008-07

Yang, J., Wang, C., Huang, K., Zhang, M., Wang, J. and Pan, X., 2020. Compound Lactobacillus sp. administration ameliorates stress and body growth through gut microbiota optimization on weaning piglets. Applied Microbiology and Biotechnology 104: 6749-6765. https://doi.org/10.1007/s00253-020-10727-4 